\title{
Non-invasive treatment options for focal cortical dysplasia (Review)
}

\author{
TING-TING WANG and DONG ZHOU

\begin{abstract}
Department of Neurology, West China Hospital, Sichuan University, Chengdu, Sichuan 610041, P.R. China
\end{abstract}
Received December 3, 2015; Accepted February 22, 2016

DOI: $10.3892 /$ etm.2016.3100

\begin{abstract}
Focal cortical dysplasia (FCD) presents a strong clinical challenge especially for the treatment of the associated epilepsy. Epilepsy in FCD is often treatment-resistant and constitutes $50 \%$ of treatment-resistant cases. Antiepileptic drugs (AEDs) have been widely used in the treatment of FCD. However, evidence to suggest their specific effect on the treatment of FCD remains to be established. In view of this resistance, several alternative treatments have been suggested. Although treatment currently involves surgical management, non-invasive treatments have been identified. The aim of the present review, was to assess non-invasive management strategies including, i) mammalian target of rapamycin (mTOR) inhibitors, ii) ketogenic diet (KD), and iii) vagus nerve stimulation (VNS). In addition, we discussed the literature available regarding the use of AEDs in FCD. Experiments conducted with mammals detailing rapamycin gene mutations in FCD have produced vital information for exploring treatment options using mTOR inhibitors. Of note is the importance of KD in children with FCD. This diet has been shown to modify disease progression by attenuating chromatin modification, a master regulator for gene expression and functional adaptation of the cell. FCD has also been studied widely with neurostimulation techniques. The outcomes of these techniques have been found to be variable. For widespread dysplasias, VNS has been shown to produce responder rates of $>50 \%$. Nevertheless, non-invasive cranial nerve stimulation techniques such as transcutaneous VNS and non-invasive VNS are gaining better patient compatibility, albeit their efficacy remains to be established.
\end{abstract}

\section{Contents}

1. Introduction

2. Pathoanatomy

Correspondence to: Dr Dong Zhou, Department of Neurology, West China Hospital, Sichuan University, 37 Guoxue Road, Chengdu, Sichuan 610041, P.R. China

E-mail: zhoudong66@yahoo.de

Key words: cortical dysplasia, epilepsy, motor inhibitors, ketogenic diet
3. Antiepileptic drugs

4. Motor inhibitors

5. Ketogenic diet

6. Composition of KD

7. Efficacy of KD

8. Vagus nerve stimulation

9. Conclusion

\section{Introduction}

Intractable epilepsy is a primary challenge in medicine especially with regard to focal cortical dysplasia (FCD). The incidence of FCD is almost $50 \%$ of all the cases of intractable epilepsy identified in adults and children. Taylor and colleagues (1) were the first to classify FCD following treatment of 10 patients with developmental malformations. Ever since its nomenclature, FCD has been subjected to re-classifications owing to ongoing advancement in neuroimaging techniques and genetic science $(1,2)$. It is useful to consider various diagnostic and treatment modalities available for FCD for an improved therapeutic outcome and treading a path towards restoring normal functioning.

Antiepileptic drugs (AEDs) have been widely used in the treatment of FCD. However, evidence to suggest their specific effect in the treatment of FCD remains to be established. In view of this resistance, several alternative treatments have been identified, particularly surgical management. Non-invasive treatments have also been considered with regard to the management of the disease. Three of these non-invasive management strategies are the focus of the current study: i) Mammalian target of rapamycin (mTOR) inhibitors, ii) ketogenic diet (KD), and iii) vagus nerve stimulation (VNS). In addition, we discussed the literature available regarding the use of AEDs in FCD. Experiments conducted with mammals detailing rapamycin gene mutations in FCD have produced vital information in exploring treatment options using mTOR inhibitors $(3,4)$. The importance of KD in children with FCD has also been identified. This diet has shown to modify disease progression by attenuating chromatin modification, a master regulator for gene expression and functional adaptation of the cell (5). FCD has been studied widely with neurostimulation techniques, the outcomes of which are variable. For widespread dysplasias, VNS has been shown to produce responder rates of $>50 \%$. Nevertheless, non-invasive cranial nerve stimulation 
techniques such as transcutaneous VNS and non-invasive VNS are gaining better patient compatibility, albeit their efficacy remains to be determined.

\section{Pathoanatomy}

Prior to proceeding to the non-invasive treatment methods, it is important to understand the pathoanatomy of FCD. FCD per se encompasses a variety of lesions such as cortical dysplasia, cortical dysgenesis or neuronal migration disorder which lead to developmental malformation. The following are the typical alterations:

i) Cortical architecture abnormalities: Columnar disorganization (formation of vertically oriented microcolumns consisting of $\geq 8$ neurons) and laminar disruption (alterations in 6-layered tangential composition of the cortex).

ii) Cytological abnormalities: hypertrophic neuron cells, outside the typical location in layer V; immature neurons, round or oval cells with large nucleus and thin rim of cytoplasm, absent in matured cortex; dysmorphic neurons, with abnormal size and morphology of axons and dendrites, as well as increased accumulation of neurofilament proteins; balloon cells, pathognomonic for Taylor-type dysplasia (initially described by Taylor in 1971) (1), usually large cells with ill-defined membrane, single or multiple eccentric nuclei and eosinophilic cytoplasm, demonstrating immunohistochemical features of neuronal and glial lineage (2).

FCD has been reclassified in various ways in the last 40 years owing to the wealth of emerging histopathological information. Currently, the scheme followed is the one suggested by Barkovich, modified recently in $2005(6,7)$, in which FCD is classified among developmental malformations in group Ib non-neoplastic malformations due to abnormal neuronal and glial proliferation or apoptosis, and IIIb malformations due to abnormal cortical organization. Prior to this development, the widely used histopathological classification $(2,8)$ bifurcated FCD into type I (benign), characterized by isolated architectural abnormality-Ia or with additional abnormal cells (such as hypertrophic cells and immature neurons)-Ib; or Type II (Taylor), encompassing larger abnormalities with dislayering and the additional presence of dysmorphic neurons (type IIa) or balloon cells (type IIb). FCD was frequently identified in hippocampal atrophy (9) or neoplastic developmental tumors [dysembryoplastic neuroepithelial tumors (DNET), ganglioglioma] or in post-traumatic and post-ischemic patients.

An attempt was also made (10) to modify the more recent Palmini classification into three types of FCD:

i) Type I, which is sub-classified into type Ia, Ib and Ic. FCD type Ia involves malformation presenting with abnormal cortical layering as a result of abnormal radial migration and maturation of neurons. FCD type Ib involves the disruption of typical six-layered tangential composition of the cortex with immature neurons. Type Ic involves compromising the two architectural abnormalities.

ii) Type II involves the malformation resulting from disrupted cortical lamination and specific cytological abnormalities. It has been sub-classified into IIa and IIb, where type IIa has dysmorphic neurons only and type IIb has dysmorphic neurons and balloon cells. iii) Type III involves malformation connected with different cortical dislamination and cytological abnormalities with the main lesion within the same area/lobe. It has been sub-classified into: Type IIIa, cortical dislayering with hippocampal atrophy; IIIb, with glial or glioneuronal tumors (DNET, ganglioglioma); IIIc, with vascular malformations (hemangiomas, arteriovenous malformations and telangiectasias); IIId, acquired at early age (trauma, ischemia or perinatal hemorrhage, infectious or inflammatory diseases) and other non-classified.

The treatment of intractable epilepsy remains largely empirical due to the lack of comparative studies showing the efficacy of multiple newer AEDs. These drugs with their mechanisms of action have opened up avenues for the possible use of their combination for better treatment outcome.

\section{Antiepileptic drugs}

In a review of a series of studies (11) conducted on drug-resistant patients, a transient responsiveness to pharmacotherapy was identified in 20 patients (17\%), which has been defined as a seizure-free period of $\geq 1$ year. In some of the patients, the seizure-free period lasted 9-12 years. One patient with dual pathology reported a seizure-free period of 28 years. Commonly used drugs in the treatment of drug-resistant epilepsy are broad spectrum AEDs such as levetiracetam, topiramate, and zonisamide for their multiple mechanisms of action.

\section{Motor inhibitors}

Medical intractability results in resistance to the conventional actions of seizure medications. However, the mechanism that causes medical intractability is unclear (11). There are distinct mechanisms involved in the initial development of the epileptic state, or epileptogenesis and for ongoing seizures. Increased neuronal excitability directly stimulates ongoing seizures. A concern regarding medical intractability and disease modification is addressed by modulating the initial signaling pathways that activate downstream mechanisms involved in epileptogenesis and seizure generation, rather than aiming the end-stage mechanisms that control neuronal excitability directly $(12,13)$. The signaling pathway involved in epileptogenic processes is the mTOR pathway, which has become the potential novel target for the treatment of epilepsy (4). This review presents evidence obtained from clinical trials and animal models to establish the antiseizure and antiepileptogenic effects of mTOR inhibitors.

The treatment of epilepsy involves the standard clinical approach. When the patient presents with seizures, therapy is initiated to prevent future seizures. This is a traditional approach and a relatively non-specific symptomatic treatment of the end-stage symptoms of epilepsy. In this form of treatment the underlying etiology or pathogenesis is not considered and the antiseizure drugs directly decrease neuronal excitability and suppress seizures in a non-specific manner (14-17). The preclinical data with regard to mTOR inhibitors are contradictory as to whether mTOR inhibitors have general anticonvulsant properties, independent of the underlying etiology. Variable and even contradictory results were 
observed in studies conducted on acute seizure animal models that assessed rapamycin for anticonvulsant effects $(3,18)$.

In one such study, the anticonvulsive effect of rapamycin was found to be mild and dependent on other parameters such as the age of the animal, the seizure model tested, and the timing of drug administration (3). A total lack of correlation between anticonvulsant efficacy and the suppression of mTOR activity was observed in the maximal electroshock seizure-threshold model. Furthermore, a longer treatment with rapamycin, which is expected to have greater mTOR inhibition, was not effective as a single dose, suggesting the non-specificity of rapamycin in terms of its anticonvulsant effects (3).

The conventional antiseizure drugs directly modulate ion channels or neurotransmitter receptors and lead to anticonvulsant effects. There is no convincing evidence that mTOR inhibitors are involved in such mechanisms. However, rapamycin regulates the protein synthesis of ion channels or other relevant proteins and may therefore be able to influence neuronal excitability (18). Those findings suggest that as an anticonvulsant the role of rapamycin is very minimal and may be effective only under particular circumstances. Thus, mTOR inhibitors may have limited clinical effectiveness as a standard, non-specific antiseizure treatment for epilepsy in general. In specific disorders such as tuberous sclerosis complex (TSC), which involve intrinsic mTOR hyperactivation mTOR inhibitors, may have stronger antiseizure efficacy. However, whether mTOR inhibitors are useful as antiepileptogenic drugs remains to be determined.

Identifying an effective antiepileptogenic drug is crucial since none of the currently available seizure medications have been demonstrated to possess antiepileptogenic or disease-modifying properties for epilepsy (14-17). Drugs possessing antiepileptogenic potential are not effective anticonvulsants, since the mechanisms of epileptogenesis are completely different from that of ictogenesis/seizure generation.

Chronic, progressive brain processes such as axonal sprouting, neuronal death, inflammation and autophagy as well as the expression of a variety of proteins mediating epileptogenesis are inhibited by mTOR inhibitors and constitute a likely candidate for possessing antiepileptogenic properties. A number of mouse models of TSC early treatment with mTOR inhibitors prior to seizure onset indicated complete prevention of the development of epilepsy. This finding may also prevent the underlying pathological, cell, and molecular defects that mediate epileptogenesis (4,19-22).

mTOR inhibitors do not correct the underlying genetic defect of TSC. Thus, discontinuation of rapamycin therapy in TSC mouse models results in the subsequent emergence of epilepsy with the associated pathological changes. mTOR inhibitors are able to prevent the underlying pathological abnormalities causing epilepsy and may qualify as a true antiepileptogenic or disease-modifying drug. This is completely different from other standard anticonvulsant drugs, which lead to only symptomatic antiseizure effects. Although rapamycin therapy is required continuously to maintain effectiveness, inhibition of the mTOR pathway is a very attractive, rational candidate as far as antiepileptogenic effects is concerned (4,19-21).

In non-TSC epilepsy animal models, there is also substantial evidence for antiepileptogenic properties. In the kainate status epilepticus model of temporal lobe epilepsy rapamycin treatment reduced the development of epilepsy and mossy fiber sprouting (12). By contrast, in the closely-related pilocarpine model only inhibition of mossy fiber sprouting was observed, whereas epilepsy was not inhibited (23). When status epilepticus was triggered electrically at the angular bundle (13) rapamycin appeared to attenuate epileptogenesis. However, a similar response was not identified following stimulation of the amygdala. Epilepsy model, species, or dose and timing of rapamycin are variable in each experimental set-up. The differences in the results are potentially attributed to these variables. Notably, the relationship between mTOR and acquired epilepsy following brain injury is not as direct and consistent as genetic epilepsy (4).

\section{Ketogenic diet}

Dietary treatments were devised for many neurological conditions such as brain tumors, Parkinsonism, Alzheimer's disease, and amyotrophic lateral sclerosis including epilepsy. The KD was widely used for the treatment of refractory epilepsy in the 1920s. It is a high fat, low carbohydrate, and protein-restricted diet. The use of KD diet decreased as it was considered difficult and unpalatable and was replaced with phenytoin and sodium valproate. Recently, there has been a resurgence in the use of KD in refractory childhood epilepsy with certain modifications such as the modified Atkins diet and the low glycemic index treatment (5).

$\mathrm{KD}$ is not widely prescribed by pediatricians and neurologists due to their lack of awareness and acceptability of KD as a treatment modality for epilepsy. Apart from this, acceptance is poor in predominantly vegetarian populations including the Chinese, dieticians are unfamiliar with the KD diet and the unavailability of labelled foods (24).

\section{Composition of KD}

$\mathrm{KD}$ is available in different combinations. The ratio of the calories varies with the age of the child. KD is a combination of protein, fats and carbohydrate with fats constituting $90 \%$ of the calories. A ratio of 4:1 $\mathrm{g}$ of fat to protein with additional carbohydrates is the standard combination, although a ratio of 3:1 may be used for younger children. Initially calories are restricted by $80-90 \%$ for age, but over time are re-adjusted to facilitate growth (25). Fluids are not restricted thereof preventing dehydration, constipation and kidney stones. KD requires strict weighing of foods and the calories have to be calculated for individual patients (5,24-30). Acceptance of classic KD is difficult especially for Chinese patients because of the dietary restrictions. Thus, a major prerequisite prior to initiation of treatment is counselling of parents and patients and a trained dietician. The diet becomes successful once treatment parental counselling has occurred, although this approach is time-consuming.

\section{Efficacy of KD}

The efficacy of KD has been previously reported. In a study on 317 Chinese children a $>50 \%$ seizure reduction was observed in $35 \%$ of the patients after 3 months, $26.2 \%$ after 6 months and $18.6 \%$ after 12 months of KD (26). Another study based on 
a systemic review reported $>50 \%$ reduction in $56 \%$ of children, $>90 \%$ reduction in $32 \%$ and complete cessation of seizures in $16 \%$ of children (27). A much larger systemic review by Keene (28) on 972 patients reported that at six months, an average of $15.6 \%$ of the patients had become seizure-free, while $33.0 \%$ were reported to have $>50 \%$ reduction in seizure frequency after commencing the diet. However, studies did not have control subjects and therefore did not yield good evidence. A randomized controlled study was performed by Neal et al (29) on children receiving KD, in which a treatment group (1-month) and a control group (4-month delay) with no changes in the antiepileptic drugs, although the patients received KD after a 1-month or 4-month delay. In 54 children in the diet group the seizure frequency after four months was significantly lower (38\% decrease in seizures) when compared to the 49 controls ( $37 \%$ increase in seizures; $\mathrm{P}<0.0001$ ) (29). A Cochrane review reports that short- to medium-term benefits in seizure control were evident following KD and these results were comparable to modern antiepileptic drugs (30). In 27 children with refractory epilepsy $48 \%$ had $>50 \%$ reduction in seizures, $15 \%$ (4 children were seizure-free at 6 months) and after a year $37 \%$ had $>50 \%$ reduction in seizures, and $18.5 \%$ ( 5 children) were seizure-free. This was a prospective study, where $55 \%$ remained on KD for 6 months, and $37 \%$ remained on KD for 1 year (24). The aforementioned studies show the efficacy of KD in the treatment of epilepsy and at least a $50 \%$ reduction in seizure frequency was observed in patients treated with KD.

Overall improvement was identified in seizure control, cognition and alertness following the use of KD. Prospective studies (5,24-30) also reported improvement in developmental quotients, attention, and social function. Various reasons are attributed to the improvement. The overall improvement of the patient may be due to control in seizure, decreased antiepileptic medication, or a non-specific effect of the diet or a combination of all these factors.

\section{Vagus nerve stimulation}

VNS is an extracranial form of neurostimulation developed before 20 years (14) that is currently used for the treatment of patients with refractory epilepsy worldwide. This form of treatment is used in patients with refractory epilepsy who are unsuitable for epilepsy surgery or in whom the benefit from such a treatment is insufficient (15). The earliest study on VNS was reported in patients with focal seizures (16). Subsequently, it was studied on other types of seizures as well as epilepsy syndromes (17). The study on focal epilepsy was comparable with the latter study. A seizure frequency reduction by $\geq 50 \%$ was observed in $1 / 3$ of patients and between a 30 and $50 \%$ reduction in seizure frequency was reported by another third of patients. Improved seizure control over time was also reported in long-term follow-up studies, although $25 \%$ of patients did not experience any positive effect even after long-term treatment by VNS (15). These results are consistent with those of antiepileptic drug trials in patients with refractory epilepsy. The principle seizure outcome with VNS studies and AEDs is the 'responder rate' (defined as the proportion of patients who have a $\geq 50 \%$ reduction in seizure frequency). Seizure-free rates are usually $<5 \%$ (31).
Engel et al suggested the Engel classification scale. This is a standard outcome scale after resective epilepsy surgery. This classification is used by most trials and case series in epilepsy surgery (32). This scale divided patients into four groups based on the outcome. Class I was patient-free of disabling seizures. This category included patients with no seizures, simple partial non-disabling seizures only, and/or a seizure-free period of $\geq 8$ months at the point of latest assessment. When patients had rare seizures at a frequency of three or less per year, they were classified as class II. A reduction in seizure frequency or seizure intensity improving the patient quality of life, was classified as class III. No reduction in seizure frequency or if the reduction was limited to such as extent that it did not improve day-to-day functioning, they were classified as class IV. The Engel classification takes into consideration seizure frequency as well as the impact of seizures on the quality of life. It has been found that $50-65 \%$ of patients report class I outcomes and approximately $80 \%$ of patients report a combination of class I and II outcomes (32).

Options are limited in patients with unresective seizure foci and failed resective surgery. Most studies report $<50 \%$ of patients having a $50 \%$ reduction in seizure frequency even with drug trials (33-35). No significant results were reported with other surgical options such as callosotomy, subpial transsections, and experimental surgical treatments such as deep brain stimulation (33-35), However, with VNS $18 \%$ had very good outcomes and another $49 \%$ showed improvement.

VNS was programmed at a significantly lower output current in patients with better clinical outcomes than patients who had a poorer outcome. A decrease in the seizure rate reduction from $36 \%$ after 3 months to $85 \%$ after 12 months of follow-up was observed in 13 patients who remained on the identical VNS settings and AED regimen (36).

A retrospective study (36-38) on a large group of patients have been useful in identifying VNS responders. However, one of the difficult issues in VNS treatment is the identification of subgroups most likely to benefit, prior to implantation. The absence of bilateral interictal epileptiform discharges was associated with a statistically significant increase in the likelihood of a seizure-free outcome. After VNS, the absence of bilateral interictal epileptiform discharges was associated with a statistically significant increase in the likelihood of a seizure-free outcome (37). Class I or II outcome was observed in a larger proportion of patients with normal intelligence. However, VNS is currently being recommended for mentally impaired patients as there is $50 \%$ chance for a class III outcome with a significant improvement in their mood and overall condition (38).

\section{Conclusion}

The treatment-resistant epilepsy in FCD is a difficult challenge for the treating neurologist. Recently, several non-invasive techniques have been used for the treatment in such cases. Of all the novel techniques, three specific forms in the form of Vagal nerve stimulation, KD and mTOR inhibitors seem to be promising due to the plethora of results showing their efficacies. Further studies are required regarding these treatment modalities to obtain more robust data so that these forms can be used for the treatment of resistant epilepsy cases of FCD. 


\section{References}

1. Taylor DC, Falconer MA, Bruton CJ and Corsellis JAN: Foca dysplasia of the cerebral cortex in epilepsy. J Neurol Neurosurg Psychiatry 34: 369-387, 1971

2. Palmini A, Najm I, Avanzini G, Babb T, Guerrini R, Foldvary-Schaefer N, Jackson G, Lüders HO, Prayson R, Spreafico R, et al: Terminology and classification of the cortical dysplasias. Neurology 62 (Suppl 3): S2-S8, 2004.

3. Hartman AL, Santos P, Dolce A and Hardwick JM: The mTOR inhibitor rapamycin has limited acute anticonvulsant effects in mice. PLoS One 7: e45156, 2012.

4. Meikle L, Pollizzi K, Egnor A, Kramvis I, Lane H, Sahin M and Kwiatkowski DJ: Response of a neuronal model of tuberous sclerosis to mammalian target of rapamycin (mTOR) inhibitors: Effects on mTORC1 and Akt signaling lead to improved survival and function. J Neurosci 28: 5422-5432, 2008.

5. Stafstrom CE and Rho JM: The ketogenic diet as a treatment paradigm for diverse neurological disorders. Front Pharmacol 3 59, 2012.

6. Barkovich AJ and Kuzniecky RI: Neuroimaging of focal malformations of cortical development. J Clin Neurophysiol 13: 481-494, 1996.

7. Barkovich AJ, Kuzniecky RI, Jackson GD, Guerrini R and Dobyns WB: A developmental and genetic classification for malformations of cortical development. Neurology 65 1873-1887, 2005.

8. Tassi L, Colombo N, Garbelli R, Francione S, Lo Russo G, Mai R, Cardinale F, Cossu M, Ferrario A, Galli C, et al: Focal cortical dysplasia: Neuropathological subtypes, EEG, neuroimaging and surgical outcome. Brain 125: 1719-1732, 2002.

9. Diehl B, Najm I, LaPresto E, Prayson R, Ruggieri P, Mohamed A, Ying Z, Lieber M, Babb T, Bingaman W, et al: Temporal lobe volumes in patients with hippocampal sclerosis with or without cortical dysplasia. Neurology 62: 1729-1735, 2004.

10. Blümcke I, Thom M, Aronica E, Armstrong DD, Vinters HV, Palmini A, Jacques TS, Avanzini G, Barkovich AJ, Battaglia G, et al: The clinicopathologic spectrum of focal cortical dysplasias: A consensus classification proposed by an ad hoc Task Force of the ILAE Diagnostic Methods Commission. Epilepsia 52: $158-174,2011$.

11. Kwan P and Brodie MJ: Refractory epilepsy: Mechanisms and solutions. Expert Rev Neurother 6: 397-406, 2006.

12. Zeng LH, Rensing NR and Wong M: The mammalian target of rapamycin signaling pathway mediates epileptogenesis in a model of temporal lobe epilepsy. J Neurosci 29: 6964-6972, 2009.

13. van Vliet EA, Forte G, Holtman L, den Burger JC, Sinjewel A, de Vries HE, Aronica E and Gorter JA: Inhibition of mammalian target of rapamycin reduces epileptogenesis and blood-brain barrier leakage but not microglia activation. Epilepsia 53: 1254-1263, 2012.

14. Penry JK and Dean JC: Prevention of intractable partial seizures by intermittent vagal stimulation in humans: Preliminary results Epilepsia 31 (Suppl 2): S40-S43, 1990.

15. Ben-Menachem E: Vagus-nerve stimulation for the treatment of epilepsy. Lancet Neurol 1: 477-482, 2002.

16. DeGiorgio CM, Schachter SC, Handforth A, Salinsky M, Thompson J, Uthman B, Reed R, Collins S, Tecoma E, Morris GL, et al: Prospective long-term study of vagus nerve stimulation for the treatment of refractory seizures. Epilepsia 41: $1195-1200,2000$

17. Holmes MD, Silbergeld DL, Drouhard D, Wilensky AJ and Ojemann LM: Effect of vagus nerve stimulation on adults with pharmacoresistant generalized epilepsy syndromes. Seizure 13: 340-345, 2004

18. Huang X, McMahon J, Yang J, Shin D and Huang Y: Rapamycin down-regulates KCC2 expression and increases seizure susceptibility to convulsants in immature rats. Neuroscience 219: 33-47, 2012.

19. Goto J, Talos DM, Klein P, Qin W, Chekaluk YI, Anderl S, Malinowska IA, Di Nardo A, Bronson RT, Chan JA, et al: Regulable neural progenitor-specific Tsc1 loss yields giant cells with organellar dysfunction in a model of tuberous sclerosis complex. Proc Natl Acad Sci USA 108: E1070-E1079, 2011.
20. Zeng LH, Rensing NR, Zhang B, Gutmann DH, Gambello MJ and Wong M: Tsc2 gene inactivation causes a more severe epilepsy phenotype than Tscl inactivation in a mouse model of tuberous sclerosis complex. Hum Mol Genet 20: 445-454, 2011.

21. Carson RP, Van Nielen DL, Winzenburger PA and Ess KC: Neuronal and glia abnormalities in Tsc1-deficient forebrain and partial rescue by rapamycin. Neurobiol Dis 45: 369-380, 2012.

22. Way SW, Rozas NS, Wu HC, McKenna J III, Reith RM, Hashmi SS, Dash PK and Gambello MJ: The differential effects of prenatal and/or postnatal rapamycin on neurodevelopmental defects and cognition in a neuroglial mouse model of tuberous sclerosis complex. Hum Mol Genet 21: 3226-3236, 2012.

23. Buckmaster PS and Lew FH: Rapamycin suppresses mossy fiber sprouting but not seizure frequency in a mouse model of temporal lobe epilepsy. J Neurosci 31: 2337-2347, 2011.

24. Sharma S, Gulati S, Kalra V, Agarwala A and Kabra M: Seizure control and biochemical profile on the ketogenic diet in young children with refractory epilepsy - Indian experience. Seizure 18: 446-449, 2009.

25. Kossoff EH, Zupec-Kania BA and Rho JM: Ketogenic diets: An update for child neurologists. J Child Neurol 24: 979-988, 2009.

26. Suo C, Liao J, Lu X, Fang K, Hu Y, Chen L, Cao D, Huang T, Li B and Li C: Efficacy and safety of the ketogenic diet in Chinese children. Seizure 22: 174-178, 2013.

27. Lefevre F and Aronson N: Ketogenic diet for the treatment of refractory epilepsy in children: A systematic review of efficacy. Pediatrics 105: E46, 2000.

28. Keene DL: A systematic review of the use of the ketogenic diet in childhood epilepsy. Pediatr Neurol 35: 1-5, 2006.

29. Neal EG, Chaffe H, Schwartz RH, Lawson MS, Edwards N, Fitzsimmons G, Whitney A and Cross JH: The ketogenic diet for the treatment of childhood epilepsy: A randomised controlled trial. Lancet Neurol 7: 500-506,2008.

30. Levy RG, Cooper PN and Giri P: Ketogenic diet and other dietary treatments for epilepsy. Cochrane Database Syst Rev 3: CD001903, 2012

31. French JA, Kanner AM, Bautista J, Abou-Khalil B, Browne T, Harden CL, Theodore WH, Bazil C, Stern J, Schachter SC, et al; Therapeutics and Technology Assessment Subcommittee of the American Academy of Neurology; Quality Standards Subcommittee of the American Academy of Neurology; American Epilepsy Society: Efficacy and tolerability of the new antiepileptic drugs II: treatment of refractory epilepsy: report of the Therapeutics and Technology Assessment Subcommittee and Quality Standards Subcommittee of the American Academy of Neurology and the American Epilepsy Society. Neurology 62: 1261-1273, 2004

32. Engel J Jr, Van Ness PC, Rasmussen TB and Ojemann LM: Outcome with respect to epileptic seizures. In: Surgical treatment of the epilepsies. Engel J Jr (ed). Raven Press, New York, NY, pp609-621, 1993.

33. Wada JA: Callosal bisection and transcallosal secondary antiepileptogenesis. Epilepsia 46 (Suppl 1): 2-6, 2005.

34. Theodore WH and Fisher RS: Brain stimulation for epilepsy. Lancet Neurol 3: 111-118, 2004.

35. Spencer SS, Schramm J, Wyler A, O'Connor M, Orbach D, Krauss G, Sperling M, Devinsky O, Elger C, Lesser R, et al: Multiple subpial transection for intractable partial epilepsy: an international meta-analysis. Epilepsia 43: 141-145, 2002.

36. Labar D: Vagus nerve stimulation for 1 year in 269 patients on unchanged antiepileptic drugs. Seizure 13: 392-398, 2004.

37. Janszky J, Hoppe M, Behne F, Tuxhorn I, Pannek HW and Ebner A: Vagus nerve stimulation: Predictors of seizure freedom. J Neurol Neurosurg Psychiatry 76: 384-389, 2005.

38. Huf RL, Mamelak A and Kneedy-Cayem K: Vagus nerve stimulation therapy: 2-year prospective open-label study of 40 subjects with refractory epilepsy and low IQ who are living in long-term care facilities. Epilepsy Behav 6: 417-423, 2005. 